\title{
Effects of diclofenac sodium on the hippocampus of rats with acute subdural hematoma: histological, stereological, and molecular approach
}

\author{
Aysın Pınar Türkmen ${ }^{1}$, Suleyman Kaplan ${ }^{1}$, Abdurrahman Aksoy², Berrin Zuhal Altunkaynak ${ }^{1}$, Kıymet Kübra Yurt ${ }^{1}$, \\ Ebru Elibol ${ }^{1}$, Cengiz Çokluk ${ }^{3}$, Mehmet Emin Onger ${ }^{1}$ \\ ${ }^{1}$ Department of Histology and Embryology, Faculty of Medicine, Ondokuz Mayıs University, Samsun, ${ }^{2}$ Department of Pharmacology, \\ Faculty of Veterinary Medicine, Ondokuz Mayıs University, Samsun, ${ }^{3}$ Department of Neurosurgery, Faculty of Medicine, Ondokuz \\ Mayıs University, Samsun, Turkey
}

\begin{abstract}
This study was aimed at evaluating the potential effects of acute subdural hematoma (ASDH) and diclofenac sodium (DS) therapy following ASDH on the rat hippocampus.

Twenty-four male Sprague Dawley rats were used and divided into four groups. $0.1 \mathrm{ml}$ of non-heparinized autologous blood from the tail vein of the animals in the non-treatment group (NTG) and treatment group (TG) was injected into the subdural space. The TG received intramuscular diclofenac sodium at a $15 \mathrm{mg} / \mathrm{kg}$ dose daily from the postoperative second hour to the seventh day after the operation. The control group (CG) and sham group (SG) were used for control and sham operations, respectively. On the postoperative eighth day, all animals were sacrificed, and the hippocampi of all animals were stereologically and histologically evaluated. Also blood samples of the animals were biochemically analyzed.

As a result of the study, the mean number of neurons in CA1, CA2, and CA3 regions of the hippocampus and the total number of neurons were decreased in the hippocampus samples of the NTG and especially the TG subjects. When comparing the second blood samples, there was no difference between the levels of adrenaline and serotonin among the groups. However, after the operation, noradrenalin levels in the treatment group were found to be higher than those of the sham and control groups $(p<0.05)$.

In the NTG and TG, histopathological findings were observed such as Nissl condensation as well as completely dead and indistinguishable neurons with abnormally shaped, shrunken cytoplasm and nuclei. Also necrotic areas on the specimens of the TG were seen. In immunohistochemical sections, c-FOS positivity was decreased in the NTG and especially the TG. Otherwise, PGC-1 $\alpha$ positive cells were increased in the NTG and especially the TG.

In this study, it was shown for the first time by means of stereological techniques that using DS after ASDH caused a decrease in the number of hippocampal neurons (CA1, CA2, and CA3 regions).
\end{abstract}

Key words: acute subdural hematoma, diclofenac sodium, hippocampus, stereology, rat. 


\section{Introduction}

Acute subdural hematoma (ASDH) is a life-threatening situation and generally leads to brain injury [16]. Brain damage is an important pathophysiological process in patients with ASDH [28]. The adverse effects of intracranial hematomas lead to several degenerative mechanisms, including ischemia-induced neuronal damage, clot-derived toxic factors, and edema formation $[26,47]$. At this point, levels of different mediators were evaluated. For example, serotonin may have protective effects on neurons [8]. Moreover, serotonin receptor agonists contribute to decrease of lesion volume and are considered as neuro-protective after ASDH [12,46]. Some researchers claim that high levels of dopamine, adrenaline, and especially noradrenaline lead to increased cerebral blood flow following brain injury $[34,35]$. Although there were mostly positive opinions, negative results were reported for high catecholamine levels. In a previous study, it was suggested that high adrenaline levels might have induced the apoptotic pathways in neonatal rats exposed to hypoxia [31]. Also, some researchers stated that high noradrenaline levels have potential for deleterious effects and the ability to induce apoptosis in PC12 cells, neuronal cells, and cardiac myocytes [6,51]. Therefore, in the current study we evaluated serotonin and catecholamine levels and attempted to detect their association with cell death.

In the treatment of cerebral hematomas, decompression was initially applied to minimize damage. Post-traumatic headache is one of the possible problems in the acute rehabilitation period for surviving patients $[38,45]$. Treatment options for the headache are non-steroidal, anti-inflammatory agents such as acetaminophen, ibuprofen, naproxen, and diclofenac sodium (DS) $[5,11]$.

We previously reported deleterious effects of DS on the sciatic nerve, hippocampal pyramidal and granular cells, and Purkinje cells of male rats following prenatal exposure $[14,32]$. Also, DS has been reported to exert a neuroprotective effect by reducing the nerve root dysfunction induced by compression [10]. Additionally, several studies showed that DS did not inhibit proliferation and differentiation of neural stem cells [25].

Although these side effects have been reported, little is known about the effect of non-steroidal anti-inflammatory drugs on the brain following the trauma caused by ASDH. The aim of the present study was therefore to investigate the effect of DS on hippocampus morphometry following ASDH and to define serotonin and catecholamine levels and their association with cell death caused by ASDH.

\section{Material and methods \\ Animals}

In this study, 24 adult male Sprague Dawley rats, weighing 300-350 g, were obtained from the Surgical Research Center. Adult rats were randomly divided into four groups, each consisting of six animals: control (CG), sham (SG), non-treatment (NTG), and treatment (TG). Following this, they were housed in standard plastic cages in a room air-conditioned to $20^{\circ} \mathrm{C}$, under a $12 / 12$-h light/dark cycle, and fed ad libitum.

One milliliter of autologous venous blood was collected from the tail vein of the animals belonging to all groups seven days before the experimental ASDH operation. No operation or drugs were given to the control (CG) group, but the SG, NTG, and TG were subjected to a surgical operation. On the day of sacrifice, all animals were taken to an adjacent room and decapitated, and blood was collected from the CG, SG, NTG and TG in polyethylene tubes containing $2 \%$ EDTA $(0.05 \mathrm{ml})$ and stored on ice until centrifuged.

For these experiments, all procedures were approved by the Animal Experiments and Ethics Committee of Ondokuz Mayıs University.

\section{Surgical preparation}

The animals in the SG, NTG, and TG were turned to a prone position, mounted on a stereotactic frame (Fig. 1A) and intraperitoneally anesthetized with urethane $1.25 \mathrm{~g} / \mathrm{kg}$. Body temperature was measured by a rectal thermometer at $37-37.5^{\circ} \mathrm{C}$ by using a heating light. Following the sagittal scalp incision, a burr hole of $3 \mathrm{~mm}$, placed $1 \mathrm{~mm}$ posterior to the coronal suture and $2 \mathrm{~mm}$ from the sagittal suture, was formed using a dental drill in the right parietal region under the microscope (Fig. 1). The incision of the dura mater was performed with a 30-gauge dental needle while in the subdural space another dental needle insertion was made. In the SG, only a burr hole was created and then the scalp was sutured with silk suture. No injection or insertion of the needle was made in the SG. The subdural hema- 

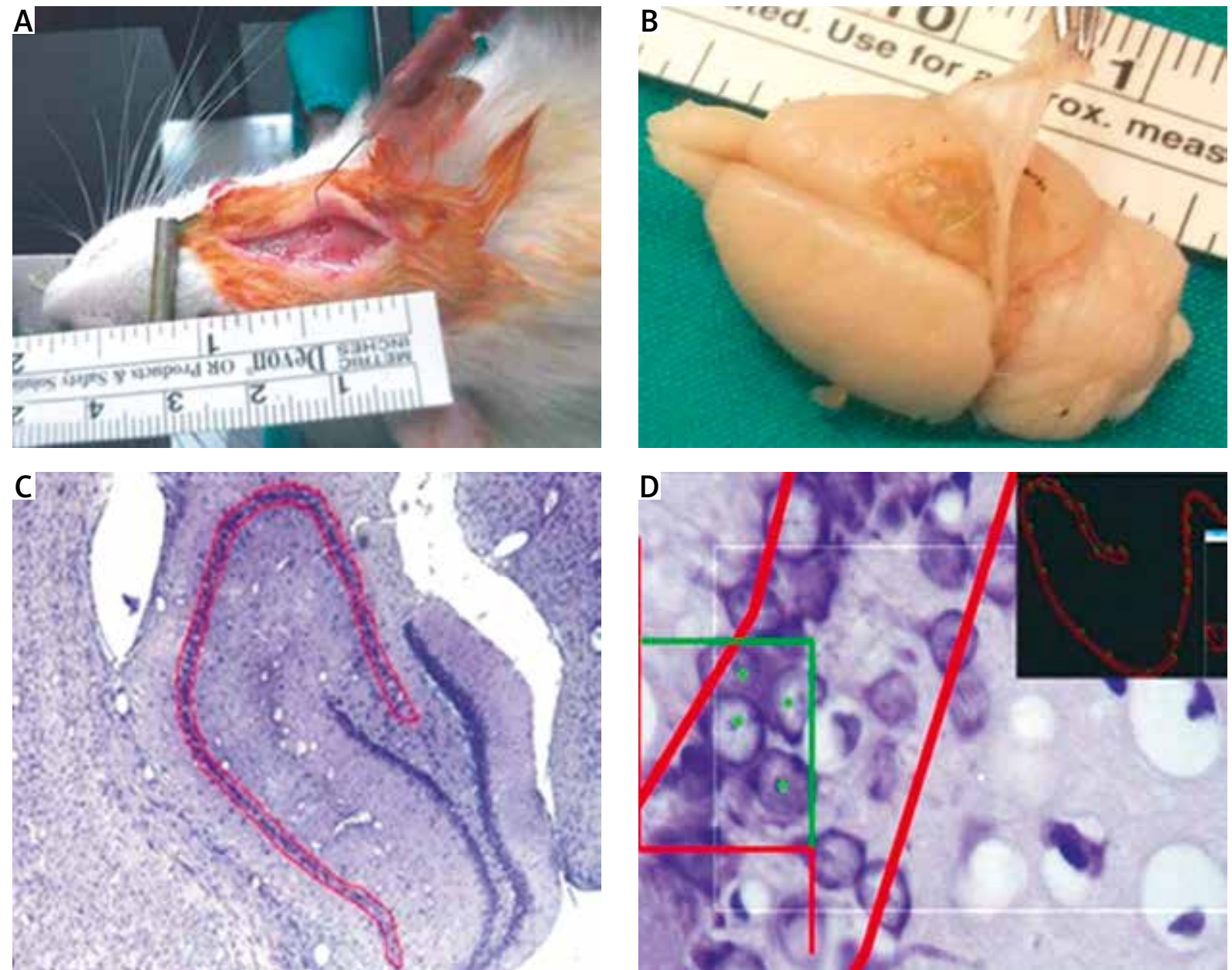

Fig. 1. Application of acute subdural hematoma model and stereological analysis procedures are shown. A) The animals were turned prone in a small animal stereotactic frame and burr-hole location; also the position of the dental needle in the subdural space is seen. B) Macroscopic appearance of the lesion. C) Lining of the hippocampus boundaries during the stereological analysis. D) Systematic random sampling and estimation of neuron numbers in the hippocampus.

toma was produced by the slow injection of $0.1 \mathrm{ml}$ non-heparinized autologous venous blood in the NTG and TG (Fig. 1A). Then, the scalp was sutured with silk suture. After waking up from anesthesia, the next seven days were spent in the normal light and dark cycle with $21 \pm 2{ }^{\circ} \mathrm{C}$ room temperature. Feed and water were supplied ad libitum [25]. The day of the operation was considered the first day of the experiment, and DS (Voltaren, $75 \mathrm{mg} / 3 \mathrm{ml}$ ampoule, Novartis, Mefar Ilaç Sanayi A.S., Kartal - Istanbul, Turkey) in a dose of $15 \mathrm{mg} / \mathrm{kg}$ daily was intramuscularly injected into the TG, beginning from the postoperative second hour to the $7^{\text {th }}$ day of the experiment. Also, no treatment was used for the NTG.

\section{Plasma collection}

On the day of the operation, blood samples were collected from the tail vein of the all animals into polyethylene tubes containing 2\% EDTA $(0.05 \mathrm{ml})$ and stored on ice until centrifuged. The first analysis of plasma levels of serotonin, adrenaline, and noradrenaline was performed in these blood samples. On the day of sacrification, after the animals were decapitated, polyethylene tubes containing 2\% EDTA $(0.05 \mathrm{ml})$ were used to collect the blood and the collected blood was kept on ice until the centrifugation. The isolation of the plasma from the whole blood was performed by centrifugation at 1300 RCF for 
25 min. and the supernatant was collected and stored at $-80^{\circ} \mathrm{C}$ until it is assayed. The second analysis of plasma levels of serotonin, adrenaline, and noradrenaline was performed in these blood samples of all the groups.

\section{High performance liquid chromatography (HPLC) for detecting plasma levels of serotonin, adrenaline, and noradrenaline}

The HPLC system consisted of multichannel pumps (LC 20AT), an autosampler (SIL 20ACHT), a degasser (DGU 20A5), a diode array detector (DAD, SPD M20A) (Shimadzu, Kyoto, Japan) and an electrochemical calorimetric array detector (Coulochem III) equipped with a model 5020 guard and 5010A dual analytical cell (ESA, Chelmsford, MA). A guard column (Inertsil ODS-3, $5 \mu \mathrm{m} \times 20 \mathrm{~mm} \times 4.0 \mathrm{~mm}, \mathrm{GL}$ Science, Tokyo, Japan) and a reversed-phase C18 column (Inertsil ODS-3V, $5 \mu \mathrm{m} \times 250 \mathrm{~mm} \times 4.6 \mathrm{~mm}$, GL Science, Tokyo, Japan) were also used. The concentrations of serotonin (5-HT), adrenaline, and noradrenaline in blood samples were determined by HPLC according to the guide of the data sheet of the kits (Eureka Serotonin and Catecolamine HPLC detection KITs, Rotakim Inc., Ankara, Turkey). The detection limit was $0.03 \mathrm{nM}$ for all three monoamines.

\section{Histological procedure}

At the end of the $7^{\text {th }}$ day, the animals were anesthetized with ketamine $(50 \mathrm{mg} / \mathrm{kg})$ and xylazine (10 $\mathrm{mg} / \mathrm{kg}$ ) intramuscularly, and neutral formalin perfusion was administered intracardially. Afterwards, the brains were removed and routinely processed and embedded in paraffin. For these experiments, all procedures were approved by the Animal Experiments and Ethics Committee of Ondokuz Mayıs University.

\section{Stereological analysis}

Hippocampal sections were taken with a microtome (Leica RM 2135, Leica Instruments, Nussloch, Germany) at $20 \mu \mathrm{m}$ thicknesses. So, from systematic random sampling was carried by $1 / 6$. Sampled sections in the cornu ammonis (CA 1-3) were collected and stained with cresyl violet. Optical dissector probes were placed in the marked area with random angles and evenly spaced. The stereology workstation consisted of a modified light microscope (Ste- reo investigator 9.0., Micro Bright Field; Colchester, USA). The pyramidal cells in counting frames and the pyramidal cells on acceptable sides were counted (Gundersen, 1986; Fig. 1C, D). It is known that 15-20 sections taken from the hippocampal region is sufficient for the estimation of the total number of neurons during the application of optical fractionator method $[17,49]$.

The total neuron number of the hippocampus was estimated by the following formula:

$$
\mathrm{N}=\Sigma \mathrm{Q} \cdot \frac{1}{s s f} \cdot \frac{1}{a s f} \cdot \frac{1}{t s f}
$$

where: $N$ - total neuron number, $\Sigma Q$ - total dissector neuron number, ssf- sectioning sampling fraction, asf - area sampling fraction, and tsf - thickness sampling fraction.

The coefficient of error (CE) and the coefficient of variation (CV) of the sampling schedule of the hippocampus were validated from a pilot study $[13,17,19]$.

\section{Immunohistochemistry \\ TUNEL labeling assay}

TUNEL (TdT-mediated dUTP-biotin nick end labeling) stain was applied to the hippocampus slices at the $5 \mu \mathrm{m}$ thickness according to the described method by Gavrieli et al. [32]. Followed by this process, the sections were treated with proteinase $\mathrm{K}$ in order to improve the antigen for $15 \mathrm{~min}$ and the sections were incubated in $3 \%$ hydrogen peroxidase solution prepared with $100 \%$ methanol to block endogenous peroxidase for five minutes. Slides were rinsed with PBS and incubated for 1 hour at $37^{\circ} \mathrm{C}$ in TdT enzyme. The samples were then washed with PBS and incubated for $30 \mathrm{~min}$ at $25^{\circ} \mathrm{C}$ with anti-digoxigenin antibody-peroxidase conjugate, rinsed with PBS, and incubated with $3,3^{\prime}$-diaminobenzidine (DAB) at $25^{\circ} \mathrm{C}$ for 5 minutes. The slides were stained in Mayer's hematoxylin. The slides were evaluated using the light microscope (Leica, LDM 4000, Wetzlar, Germany).

\section{Immunohistochemical staining of c-FOS and PGC-1 $\alpha$}

Paraffin-embedded hippocampus samples were also used for c-FOS and PGC-1 $\alpha$ immunohistochemistry. The deparaffinization of tissue sections of $5 \mu \mathrm{m}$ was done in xylene and they were rehydrated in ethanol and in water and phosphate-buffered saline 
respectively. The blockage of endogenous peroxidase was performed by immersion in 3\% hydrogen peroxide. The tissue sections were then incubated with c-FOS and PGC- $1 \alpha$ antibodies (Dako, Istanbul, Turkey) at a concentration of $5 \mu \mathrm{g} / \mathrm{ml}$ for $1 \mathrm{~h}$ at room temperature. Control sections were incubated with phosphate-buffered saline containing normal goat serum without a primary antibody. Immunostaining was then detected with a streptavidin-biotin complex kit (Dako) and developed with a di-amino benzidine tetrahydrochloride kit. The sections were counterstained with Mayer's hematoxylin followed by light microscopy.

\section{Statistical analysis}

Microsoft SPSS version 15.0 for Windows was used for statistical analyses. One-way ANOVA (Bonferroni post-hoc test) was applied to compare the results of the groups. All statistical values under 0.05 were considered significant.
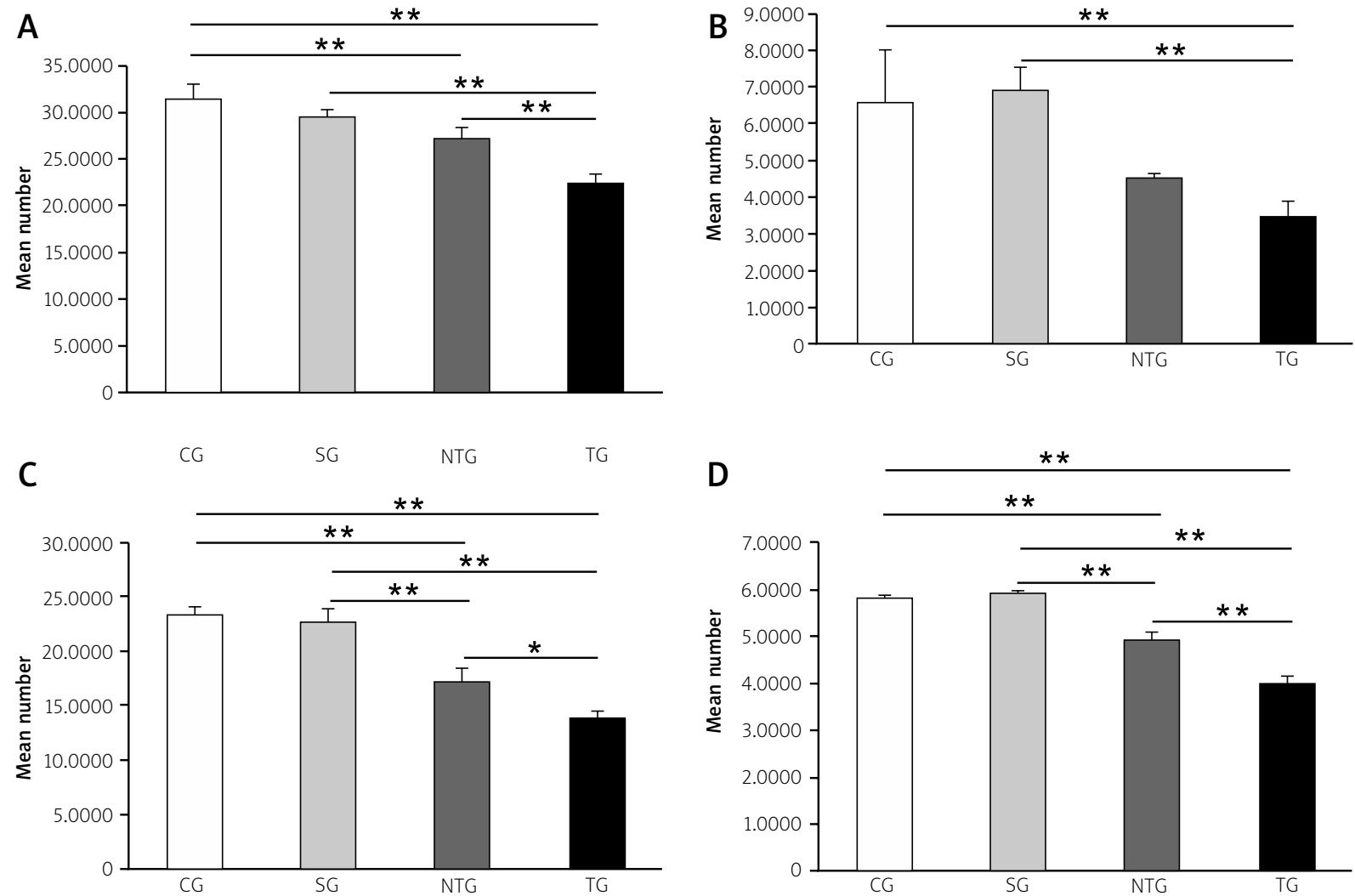

Fig. 2. Mean numerical densities of pyramidal neurons in CA1, CA2 and CA3 area of hippocampus and total number of neurons in hippocampus among CG, SG, NTG and TG are shown in A, B, C and D, respectively. ${ }^{*} p<0.05,{ }^{* *} p<0.01($ mean \pm SEM). 
A
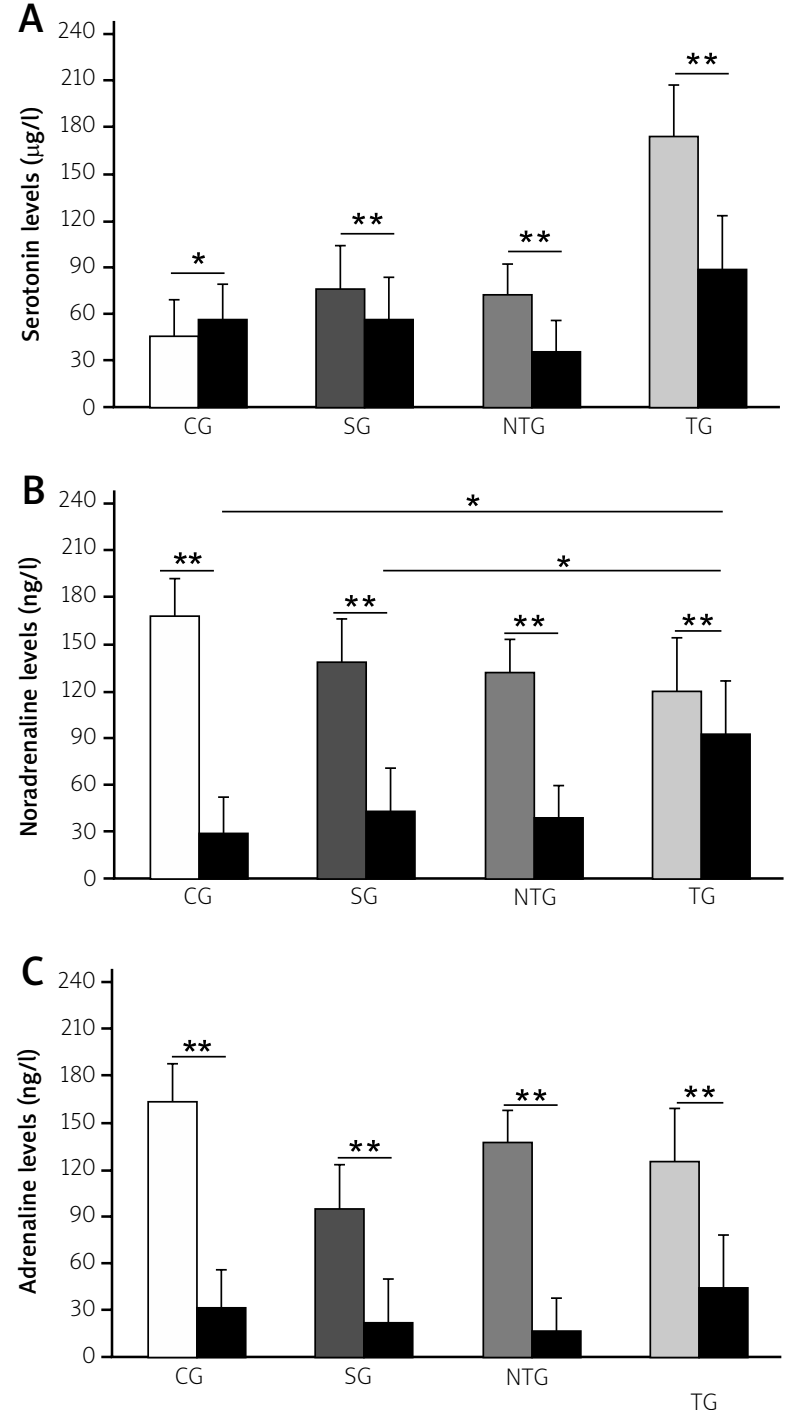

Fig. 3. Mean pre- and post-operative plasma levels of serotonin, noradrenaline and adrenaline among CG, SG, NTG and TG are shown in A, B and C, respectively. ${ }^{*} p<0.05,{ }^{\star *} p<0.01$ (mean \pm SEM).

\section{Biochemical results}

\section{Plasma serotonin levels}

When comparing the first and postoperative serotonin levels, it was found that they were significantly reduced after the operation in the SG $(p<0.05)$ and especially in the NTG and TG $(p<0.01)$. In the control group, postoperative plasma serotonin levels significantly increased in comparison to initial levels $(p<0.05)$. However, there was no significant difference in the postoperative plasma levels of serotonin among the groups (Fig. 3A, $p>0.05$ ).

\section{Plasma noradrenaline levels}

Postoperative plasma levels of noradrenaline were significantly increased after the operation, especially in the TG as compared to the other groups $(p<0.05)$. On the other hand, the plasma noradrenaline levels were significantly decreased in all groups at the second analysis (Fig. 3B, $p<0.01$ ).

\section{Plasma adrenaline levels}

After the operation, plasma adrenaline levels were significantly lower than pre-operative ones in all groups $(p<0.01)$. Among the second adrenaline levels of the groups, no significant difference was found (Fig. 3C, $p>0.05$ ).

\section{Light microscopic results}

\section{Results of the control (CG) and sham (SG) group}

In sections obtained from the hippocampus of rats among the CG and SG, a conventional healthy structure of the hippocampus was observed at the light microscopic level. The shape of neurons was pyramidal in the hippocampus. Furthermore, these neurons had euchromatic nuclei. The neurons and their extensions were normal. Histological structure of the CG that represented a healthy appearance is shown in Figure 4.

\section{Results of the non-treatment group (NTG)}

In the NTG, neurons included heterochromatic, pycnotic nuclei (Fig. 5, white asterisk) and darkstained cytoplasm (Fig. 5, black-thick arrowhead). Moreover, angular displacement of pyramidal neurons was observed in the hippocampus sections of the NTG (Fig. 5, white arrow). We also examined neurons with a dark and shrunken cytoplasm, and their nuclei had some vacuoles (Fig. 5A and C). Abnormally shaped oligodendrocytes, microglia, and astrocytes were also observed (Fig. 5, white arrowhead). Trunks of the primary dendrites were dilated and pale stained compared to those in the other groups (Fig. 5, black-thin arrowhead). Chromatolysis was detected in degenerated neurons in the hippocampus samples of this group (Fig. 5, black arrow). Moreover, in hippocampal slices of the NTG, some neurons were at the initial stage of degeneration, but some of the neurons were fully degenerated or dead. 


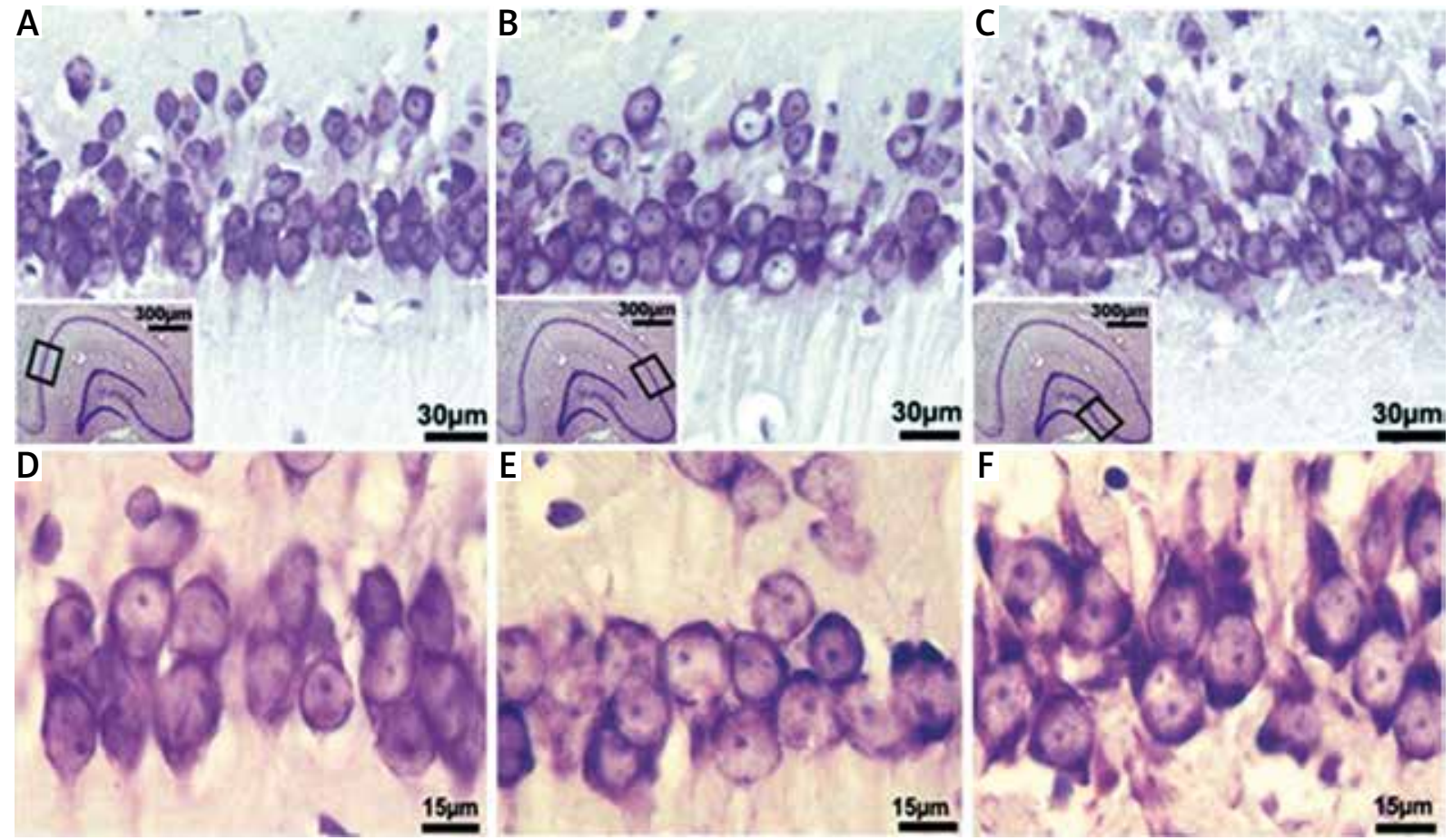

Fig. 4. Light microscopic sections obtained from the hippocampus of the control group. In A-C and D-F, specified portions of CA1, CA2, and CA3 regions are observed at low and high magnification, respectively. Thumbnails in the framed areas show hippocampal regions at the lowest magnification. In D-F, healthy neurons are clearly seen in CA regions. Cresyl violet.
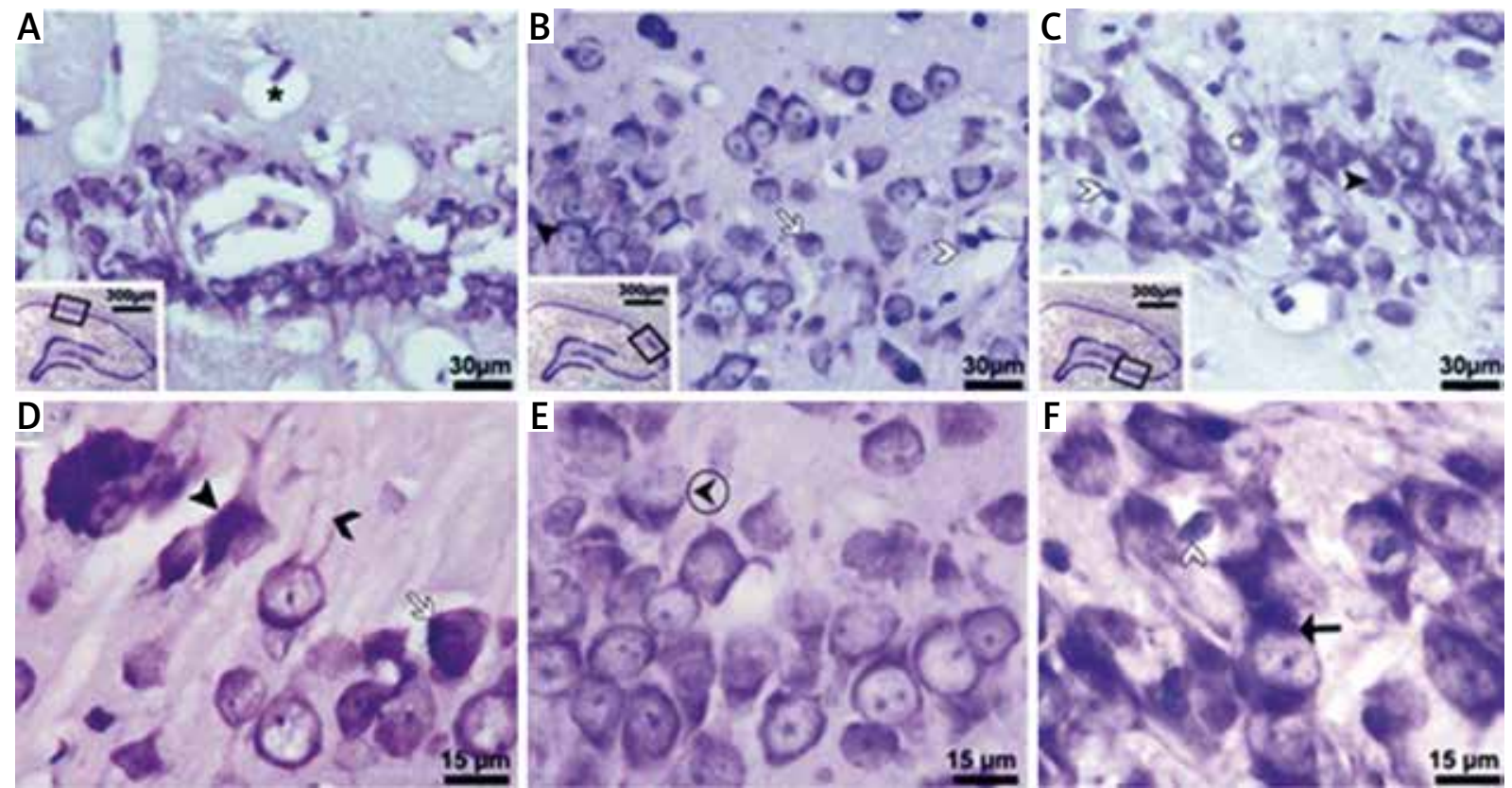

Fig. 5. Light micrographs obtained from the hippocampi of NTG. White asterisk - pycnotic neurons; white arrows - neurons with dark-stained cytoplasm; white arrowheads - glia cells; thick-black arrowhead neurons with indistinguishable cytoplasm and nucleus; black asterisk - vacuole; black ringed arrowhead - swelled nucleus with pale chromatin; thin black arrowhead - enlarged and pale stained trunks of primary dendrites; black arrows - concentrated Nissl granule containing areas. Cresyl violet. 

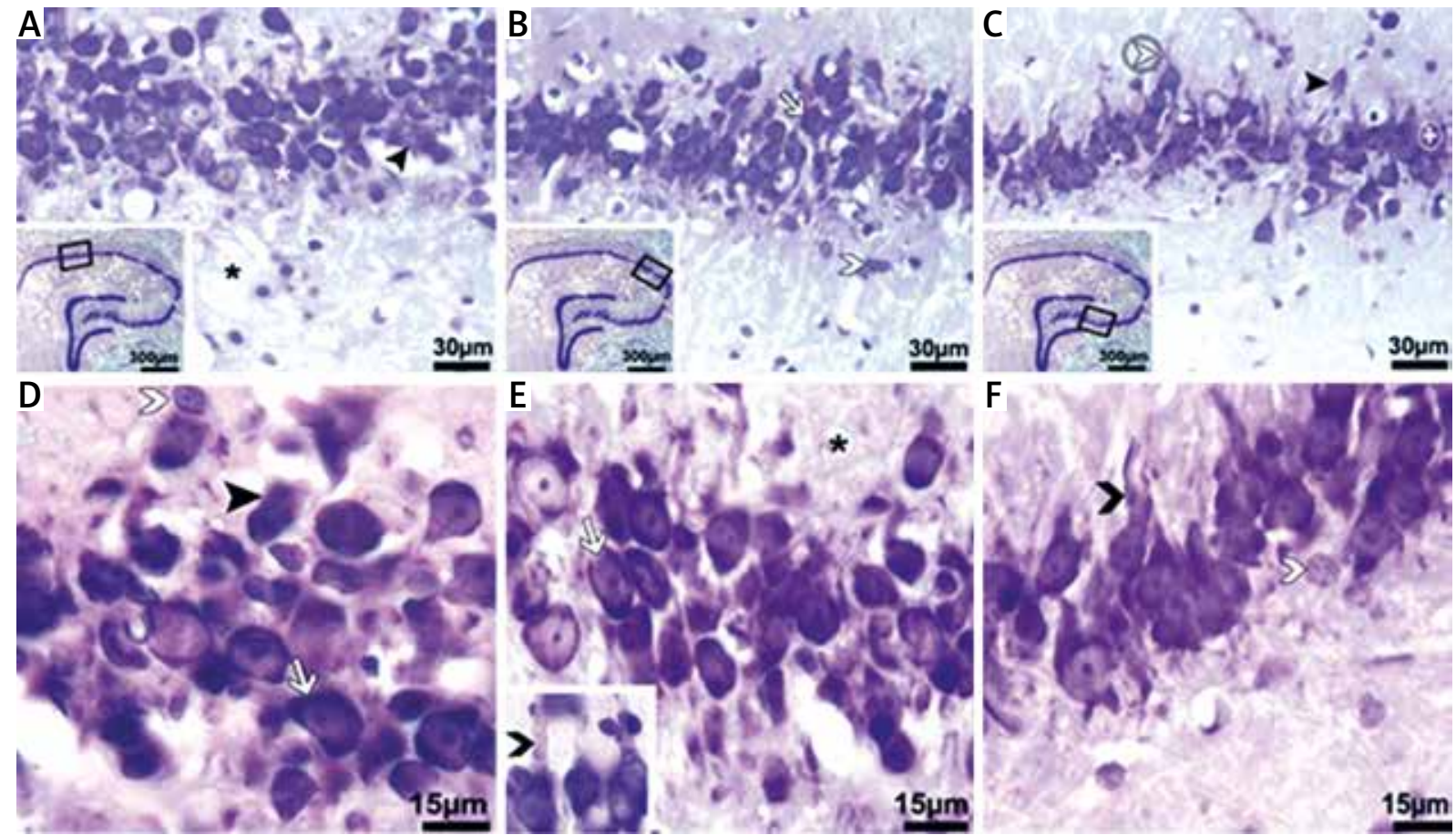

Fig. 6. Light micrographs obtained from the hippocampi of TG. White asterisk - pycnotic neurons; white arrows - neurons with dark-stained cytoplasm; white arrowhead - glia cells; white-ringed arrowhead wavy appeared axon terminals; white ringed plus - necrotic area; thick-black arrowhead - neurons with indistinguishable cytoplasm and nucleus; black asterisk - vacuole; thin-black arrowhead - enlarged and pale stained trunks of primary dendrites. Cresyl violet.

\section{Results of the treatment (TG) group}

In the light microscopic sections that were obtained from the TG, axonal extension was observed such that trunks of the primary dendrites were enlarged and pale stained in comparison to those of the CG (Fig. 6, thin-black arrowhead). Also, in the peripheral part of some degenerated neurons, Nissl condensation occurred, meaning that chromatolysis was defined (Fig. 6; black arrow). Some neurons are indistinguishable with their pale nuclei and swollen cytoplasm (Fig. 6, black ring arrowhead). In addition to neurons at the early stage of cellular damage, completely dead and indistinguishable neurons with abnormally shaped, shrunken cytoplasm and nuclei were also found (Fig. 6, thick black arrowhead). In white matter, edema, degenerated axon remains, and micro-vacuolization were observed (Fig. 6, black asterisks).

Some structures were observed as necrotic areas on certain specimens (Fig. 6, white ring + sign). It is noteworthy that chromatin in the nucleus was decreased, and dark-stained cytoplasm of pyramidal neurons was detected (Fig. 6, white arrow). Furthermore, the axons of pyramidal neurons were wavy in appearance (Fig. 6, white ring arrowhead).

\section{Immunohistochemical results}

No positive cell was seen in TUNEL-stained hippocampus sections of all the groups (Fig. 7). In the control and sham groups, neurons in the hippocampus were well stained with c-FOS, and immune reactivity was decreased following the ASDH procedure and especially DS treatment (Fig. 8C and D). However, in the NTG and TG, positivity of PGC-1 $1 \alpha$ was increased after ASDH operation and especially DS treatment (Fig. 9 C and D).

\section{Discussion}

In recent years, despite improvements in the imaging and treatment modalities used in the diagnosis of head trauma, related morbidity and mortality are still high [11]. Traumatic brain injury may lead to serious complications such as intracranial 

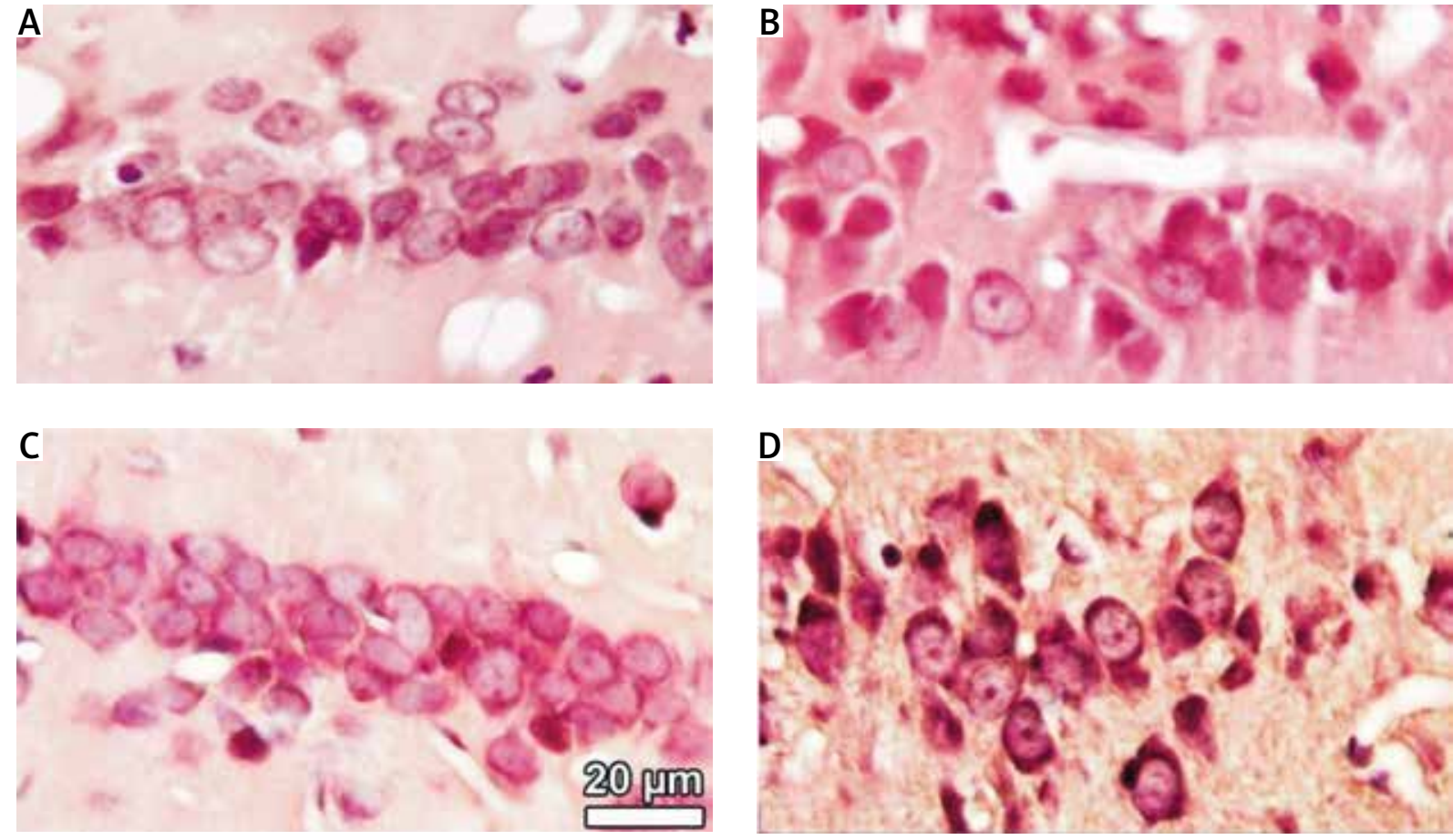

Fig. 7. Light micrographs showing TUNEL immune reactivity at 40× magnification for CG (A), SG (B), NTG (C) and TG (D). No TUNEL positivity was seen in the groups. For all micrographs, magnification bars are the same, with $20 \mu \mathrm{m}$.

hematomas. Subdural hematomas constitute 50 to $60 \%$ of traumatic intracranial hematomas [52]. The most frequent symptom of acute subdural hematoma is the sudden onset of severe headaches. Diclofenac sodium (sodium-(o-[(2,6-dichlorophenyl)amino]-phenyl)-acetate) (DS) is a non-steroidal, antiinflammatory drug characterized by a relatively low molecular weight $[25,41]$. It has a role in inhibiting the cyclooxygenase (COX) enzyme, reducing arachidonic acid release and improving its uptake [41]. It is a strong analgesic and has an antipyretic role. Hence, it is commonly used for treatment of headaches caused by traumatic brain injury. In this study, we aimed to detect the effect of ASDH and DS treatment on the hippocampus.

In the literature, a number of studies of traumatic brain injury have been conducted in animal models, and fluid percussion, vacuum deformation, and weight reduction methods were used [43]. Since these methods can lead to severe, primary diffuse brain damage, Sasaki and Dunn's model was used in our study [39]. So, the model is specific for presenting the effects of ASDH.
Also, experimental animal models of traumatic brain injury have generally focused on necrotic and apoptotic neuronal cell death. As a result of the studies, axonal damage, memory, and learning defects were reported [20,21]. According to Hausmann et al., following the brain injury, neurons are subjected to apoptotic cell death. However, in our study we detected apoptosis by TUNEL stain after the ASDH in neither the NTG nor the TG. Therefore, we suspected that occurrence of cell death after ASDH may have been caused by ischemic necrosis. However, several papers have suggested that c-FOS-triggered AP-1 might mediate apoptosis through transcriptional regulation of expression of the FasL gene to start the extrinsic apoptotic pathway [23, 24]. Our data indicate that c-FOS may be related to neuronal proliferation, because we did not detect increasing c-FOS immune reactivity or an apoptotic signal with the TUNEL stain following ASDH in either the NTG or the TG. Also, we thought that c-FOS immune reactivity decreased following ASDH because of the decreased cellular activity. Our data on PGC-1 $\alpha$ immune reactivity indicated that PGC-1 $\alpha$ positivity was higher in the NTG and TG than in the control and sham groups. 

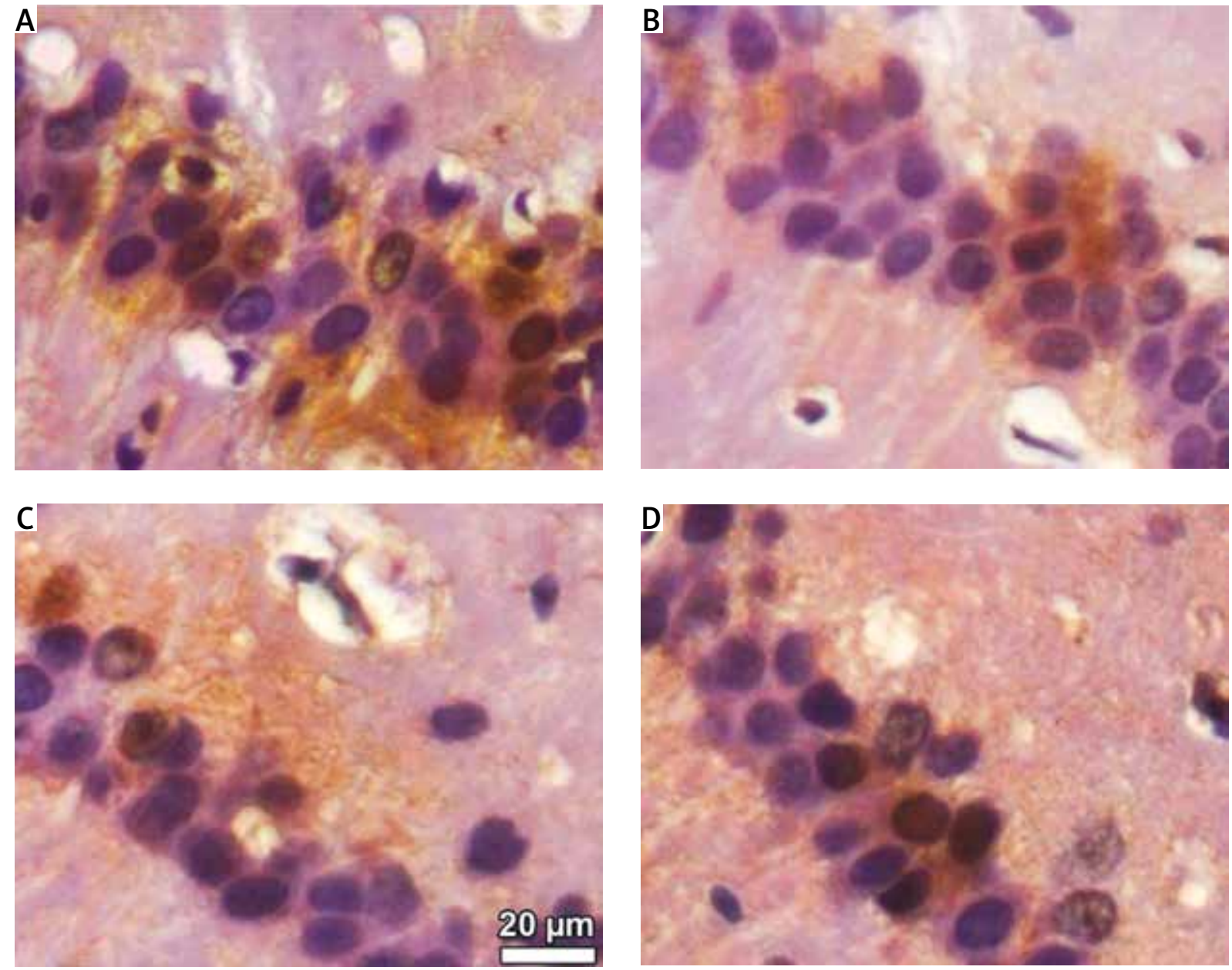

Fig. 8. Light micrographs showing c-FOS immune reactivity at 40× magnification for CG (A), SG (B), NTG (C) and TG (D). Particularly, C-FOS positive cells were noted in CG and SG. These positive cells were decreased in NTG and especially TG. For all micrographs, magnification bars are the same, with $20 \mu \mathrm{m}$.

Therefore, we suggest that PGC-1 $\alpha$-mediated upregulation of the antioxidant defense system could promote cell death following ASDH.

In the study of Tran et al. (2006), a lateral perfusion model was used, and they studied the contralateral hippocampus area using the optical fractionator method seven days after brain injury. According to their results, the number of neurons significantly decreased compared to that of the sham group [48].

According to our findings, the number of neurons in the CA1 region of the NTG was significantly decreased at seven days after ASDH $(p<0.01)$. The number of neurons in the CA2 region showed no significant change compared to the control group ( $p=1.135)$, because the CA2 region is variably sized, and generally no significant difference could be determined between study groups in terms of the number of neurons in this region [36]. Like in the CA1 region, the number of neurons in the CA3 region of the NTG was significantly reduced compared to the control group $(p<0.01)$. When we evaluated the total number of neurons, it was found to be significantly lower in the NTG than the control group $(p<0.01)$. In the sham group, the number of neurons in the CA3 region was significantly different than the number in the control group $(p<0.01)$. This reduction in the number of neurons of the subjects in the sham group may be caused by stress during surgical procedures and skull defects [44]. Indeed, in a previous study, saline injection stress was shown to reduce the number of neurons [3]. 

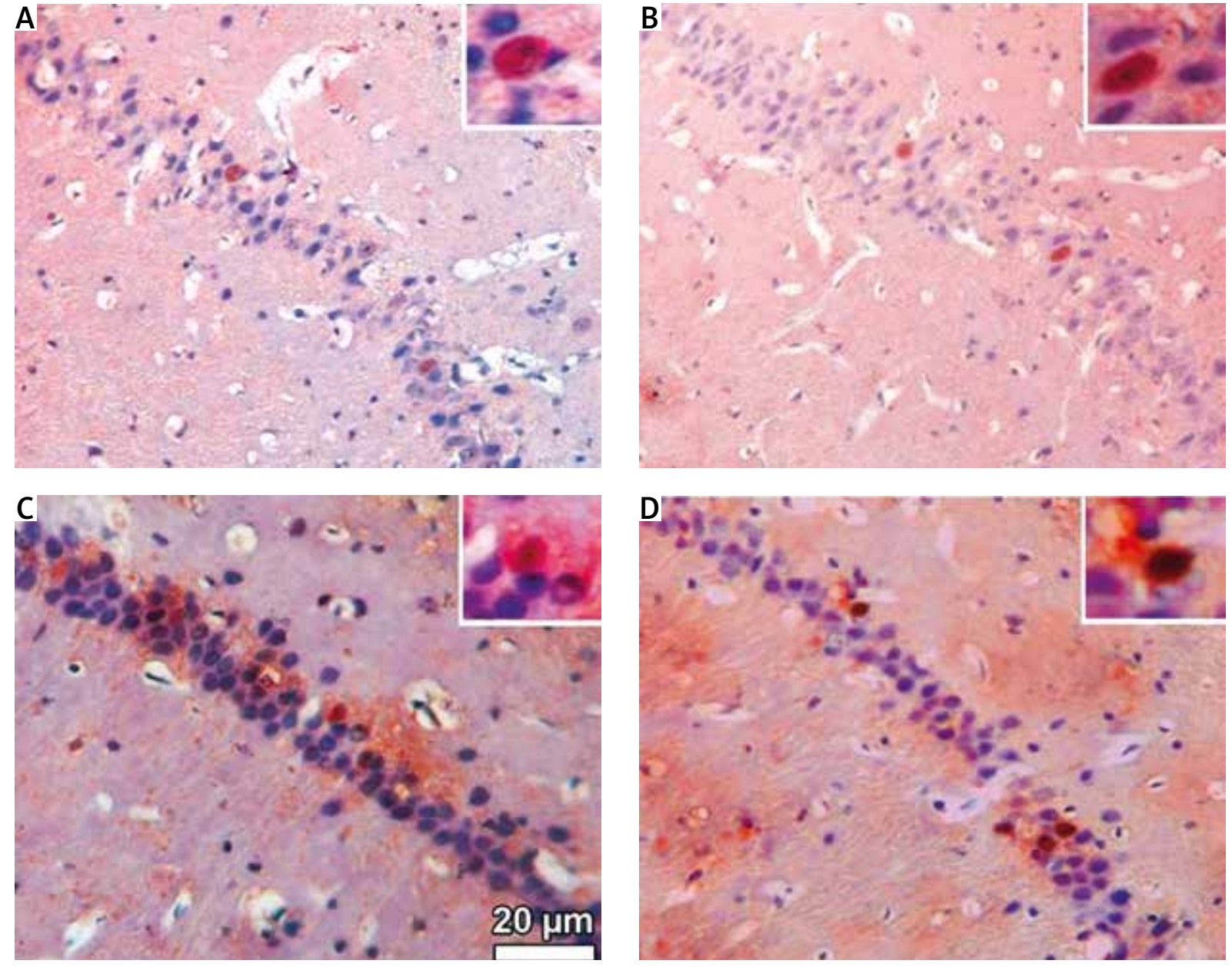

Fig. 9. Light micrographs showing PGC-1 $\alpha$ immune reactivity at $40 \times$ magnification for CG (A), SG (B), NTG (C) and TG (D). PGC-1 $\alpha$ positivity was increased in NTG and especially TG. Small pictures show PGC-1 $\alpha$-positive neurons at $\times 100$ magnification in all groups. For all micrographs, magnification bars are the same, with $20 \mu \mathrm{m}$.

Adverse effects of DS on the CNS have been clearly shown [3,33]. DS inhibits the differentiation and proliferation of neural cells in the hippocampus [14]. In the literature, 20-week-old male rats that were prenatally exposed to DS exhibited a reduction in the number of hippocampal neurons $[14,18]$. As a result of analysis by our stereological findings, DS treatment caused a decrease in the number of neurons compared to control and sham groups $(p<0.01)$. However, DS treatment in the TG did not cause significant changes in the number of neurons as compared to the NTG $(p>0.05)$.

In our study, histopathological findings were similar to the findings of ischemia and necrosis [4]. In our histological examination of the hippocampus after acute unilateral hematoma, neuronal shrink- age, pycnotic neurons, and vacuolar degenerations were detected in the NTG. Also, DS application increased the degeneration of the neurons, and more neuron death was seen in the TG than in the NTG. Also DS treatment caused a decrease of the c-FOS and PGC- $1 \alpha$ activity. Therefore cellular loss was higher in the TG than that of the NTG.

Previous studies of acute stress showed an increased rate of serotonin metabolism in the brain. The lower level of serotonin that results is not helpful in overcoming stressful situations in the future. This reduction leads to changes in sleep patterns and eating habits, and impairs sensitivity to pain [22]. In previous studies, low serum levels of serotonin have been identified in migraine patients [30]. In our study, the first measurements of the serotonin levels showed 
large differences, because the control group was not subjected to the operation on the operation day, so the level was low in that group. Because the TG was subjected to the operation and drug injection, it had the highest level. Serotonin levels of the other two groups were approximately the same as each other, higher than those of the controls, but lower than that of the TG. This situation resulted from their stress level being between the CG and TG. When comparing the second plasma levels of serotonin, a significant difference was found between the first and second levels of the groups. Also, the second serotonin levels were significantly reduced after the operation in the NTG and TG $(p<0.01)$. This reduction may be due to an inability to cope with the physical stress of the operation [22].

Stress may cause an increase of noradrenaline and adrenaline [27], but DS application may cause a reduction in stress in the TG. The second plasma levels of noradrenaline were found to be significantly increased in the TG in comparison to those of the other groups. In addition, adrenaline levels were increased in comparison to those of the other groups (not significant).

In conclusion, the results presented here show cell loss in the hippocampus due to exposure to ASDH by using stereological methods. Moreover, after ASDH, DS treatment caused a further increase in cell loss. Therefore, further studies are required to confirm this and to refine the effect mechanism and treatment alternatives to DS so as to decrease pain and prevent cell loss in patients with ASDH.

\section{Acknowledgments}

This study was supported by Scientific Research Foundation of Ondokuz Mayıs University (PYO.TIP.1904. 12.028).

\section{Disclosure}

Authors report no conflict of interest.

\section{References}

1. Altunkaynak BZ, Ozbek E, Unal B, Aydın N, Aydın MD, Vuraler O. Chronic treatment of haloperidol induces pathological changes in striatal neurons of guinea pigs: a light and electron microscopical study. Drug Chem Toxicol 2012; 35: 406-417.

2. Altunkaynak BZ, Unal D, Altunkaynak ME, Halici Z, Kalkan Y, Keles ON, Aksak S, Selli J, Unal B. Effects of diabetes and ovariectomy on rat hippocampus (a biochemical and stereological study). Gynecol Endocrinol 2012; 28: 228-261.
3. Aygün D, Kaplan S, Odaci E, Onger ME, Altunkaynak ME. Toxicity of non-steroidal anti-inflammatory drugs: a review of melatonin and diclofenac sodium association. Histol Histopathol 2012; 27: 417-453.

4. Brierley JB, Meldrum BS, Brow AW. The threshold and neuropathology of cerebral "anoxic-ischemic" cell change. Arch Neurol 1973; 29: 367-374.

5. Burdan F, Starosławska E, Szumiło J. Prenatal tolerability of acetaminophen and other over-the-counter non-selective cyclooxygenase inhibitors. Pharmacol Rep 2012; 64: 521-528.

6. Burke WJ, Schmitt CA, Miller C, Li SW. Norepinephrine transmitter metabolite induces apoptosis in differentiated rat pheochromocytoma cells. Brain Res 1997; 760: 290-293.

7. Canan S, Aktaş A, Ulkay MB, Colakoglu S, Ragbetli MC, Ayyildiz M, Geuna S, Kaplan S. Prenatal exposure to a non-steroidal antiinflammatory drug or saline solution impairs sciatic nerve morphology: a stereological and histological study. Int I Dev Neurosci 2008; 26: 733-738.

8. Cifariello A, Pompili A, Gasbarri A. 5-HT receptors in the modulation of cognitive processes. Behav Brain Res 2008; 195: 171-179.

9. Communal C, Singh K, Pimentel DR, Colucci WS. Norepinephrine stimulates apoptosis in adult rat ventricular myocytes by activation of the $\beta$-adrenergic pathway. Circulation 1998; 98: 13291363.

10. Cornefjord M, Olmarker K, Otani K, Rydevik B. Effects of diclofenac and ketoprofen on nerve conduction velocity in experimental nerve root compression. Spine (Phila Pa 1976) 2001; 26: 21932200.

11. Crooks CY, Zumsteg JM, Bell, KR. Traumatic brain injury: a review of practice management and recent advances. Phys Med Rehabil Clin N Am 2007; 18: 681-710.

12. Dooley AE, Pappas IS, Parnavelas JG. Serotonin promotes the survival of cortical glutamatergic neurons in vitro. Exp Neurol 1997; 148: 205-214.

13. Dursun H, Albayrak F, Uyanik A, Keleş NO, Beyzagül P, Bayram E, Halici Z, Altunkaynak ZB, Süleyman H, Okçu N, Ünal B. Effects of hypertension and ovariectomy on rat hepatocytes Are amlodipine and lacidipine protective? (A stereological and histological study). Turk J Gastroenterol 2010; 21: 387-395.

14. Gokcimen A, Rağbetli MC, Baş O, Tunc AT, Aslan H, Yazici, AC, Kaplan S. Effect of prenatal exposure to an anti-inflammatory drug on neuron number in cornu ammonis and dentate gyrus of the rat hippocampus: a stereological study. Brain Res 2007; 1127: 185-192.

15. Graham DI, McIntosh TK, Maxwell WL, Nicoll JA. Recent advances in neurotrauma. J Neuropathol Exp Neurol 2000; 59: 641-651.

16. Greenberg MS. Head trauma. In: Handbook of Neurosurgery. Thieme, New York 2001; 626-686.

17. Gundersen HJ, Jensen EB. The efficiency of systematic sampling in stereology and its prediction. J Microsc 1987; 147: 229-263.

18. Gurtskaia G, Tsiklauri N, Nozadze I, Nebieridze M, Tsagareli MG. Antinociceptive tolerance to NSAIDs microinjected into dorsal hippocampus. BMC Pharmacol Toxicol 2014; 15: 10.

19. Güven D, Altunkaynak BZ, Ayranci E, Kaplan S, Bildircin ED, Kesim Y, Raqbelti MC. Stereological and histopathological evaluation of ovary and uterine horns of female rats prenatal- 
ly exposed to diclofenac sodium. J Obstet Gynaecol 2013; 33 258-321.

20. Hausmann R, Biermann T, Wiest I, Tübel J, Betz P. Neuronal apoptosis following human brain injury. Int I Legal Med 2004; 118: 32-36.

21. Hensler JG. Handbook of the Behavioral Neurobiology of Serotonin 2010; 21: 367-378.

22. Jesberger JA, Richardson JS. Neurochemical aspects of depression: The past and the future? Int J Neurosci 1985; 27: 19-47.

23. Kasibhatla S, Brunner T, Genestier L, Echeverri F, Mahboubi A, Green DR. DNA damaging agents induce expression of Fas ligand and subsequent apoptosis in T lymphocytes via the activation of NF-kappa B and AP-1. Mol Cell 1998; 4: 543-551.

24. Kolbus A, Herr I, Schreiber M, Debatin KM, Wagner EF, Angel P. $C$-Jun-dependent CD95- $L$ expression is a rate-limiting step in the induction of apoptosis by alkylating agents. Mol Cell Bio 2000; 20: 575-582.

25. Kudo C, Kori M, Matsuzaki K, Yamai K, Nakajima A, Shibuya A, Niwa H, Kamisaki Y, Wada K. Diclofenac inhibits proliferation and differentiation of neural stem cells. Biochem Pharmacol 2003; 66: 289-295.

26. Lacut K, Bressollette L, Le Gal G, Etienne E. VICTORI Ah (Venous Intermittent Compression and Thrombosis Occurrence Related to Intra-cerebral Acute hemorrhage) Investigators Prevention of venous thrombosis in patients with acute intracerebral hemorrhage. Neurology 2005; 65: 865-874.

27. Manunta Y, Edeline JM. Noradrenergic induction of selective plasticity in the frequency tuning of auditory cortex neurons. J Neurophysiol 2004; 92: 1445-1463.

28. McClelland S, Mackey SJ, Kim SS. The dangerous gamble of heparinization within two weeks of non operative traumatic acute subdural hematoma in patients with increased stroke risk: a case series. J Postgrad 2014; 60: 194-201.

29. Merens W, Vander Does AJW, Spinhoven P. The effects of serotonin manipulations one motional information processing and mood. J Affect Disord 2007; 103: 43-62.

30. Nagata E, Shibata M, Hamada J, Shimizu T, Katoh Y, Gotoh K, Suzuki N. Plasma 5-hydroxytryptamine (5-HT) in migraine during an attack-free period. Headache 2006; 46: 592-596.

31. Noh JS, Kim EY, Kang JS, Kim HR, Oh YJ, Gwag BJ. Neurotoxic and neuroprotective actions of catecholamines in cortical neurons. Exp. Neurol 1999; 159: 217-241.

32. Odaci E, Cihan OF, Aslan H, et al. Prenatal diclofenac sodium administration increases the number of Purkinje cells in female rats: a stereological study. Int I Dev Neurosci 2010; 28: 145-196.

33. Ozyurt B, Kesici H, Alıcı SK, Yılmaz S, Odacı E, Aslan H, Rağbetli MÇ, Kaplan S. Prenatal exposure to diclofenac sodium chang es the morphology of the male rat cervical spinal cord: A stereological and histopathological study. Neurotoxicol Teratol 2011; 33: 282-287.

34. Patel NJ, Chen MJ, Russo-Neustadt AA. Norepinephrine and nitric oxide promote cell survival signaling in hippocampal neurons. Eur J Pharmacol 2010; 10: 633-636.

35. Pfister D, Strebel SP, Steiner LA. Effects of catecholamines on cerebral blood vessels in patients with traumatic brain injury. Eur J Anaesthesiol Suppl 2008; 42: 98-103.
36. Rağbetli MC, Aydinlioğlu A, Koyun N, Yayici R, Arslan K. Total neuron numbers in CA1-4 sectors of the dog hippocampus. Indian J Med Res 2010; 131: 780-785.

37. Ragbetli MC, Ozyurt B, Aslan H, Odaci E, Gokcimen A, Sahin B, Kaplan S. Effect of prenatal exposure to diclofenac sodium on Purkinje cell numbers in rat cerebellum: a stereological study. Brain Res 2007; 1174: 130-135.

38. Safaz I, Alaca R, Yaşar E, Tok F, Yilmaz B. Medical complications, physical function and communication skills in patients with traumatic brain injury: a single center 5 -year experience. Brain Inj 2008; 22: 1-7.

39. Sasaki M, Dunn L. A model of acute subdural hematoma in the mouse. J Neurotrauma 2001; 18: 1241-1246.

40. Schmitz C. Variation of fractionator estimates and its prediction. Anat Embryol 1998; 198: 371-397.

41. Scholer DW, Ku EC, Boettcher I, Schweizer A. Pharmacology of diclofenac sodium. Am J Med 1986; 80: 34-46.

42. Simon E, Bánk J, Gál J, Siro P, Novak L, Fulesdi B, Molnarc C. Administration of preemptive analgesia by diclofenac to prevent acute post craniotomy headache. Iddeggyogy Sz 2012; 65: 302-306.

43. Siu SSN, Yeung JHK, Lau TK. A study on placental transfer of diclofenac in first trimester of human pregnancy. Hum Reprod 2000; 15: 2423-2425.

44. Sorrells SF, Sapolsky RM. An inflammatory review of glucocorticoid actions in the CNS. Brain Behav Immun 2007; 21: 259-272.

45. Stalnacke BM. Post concussion symptoms in patients with injury-related chronic pain. Rehabil Res Pract 2012; 528: 265.

46. Sun ZL, Feng DF. Biomarkers of cognitive dysfunction in traumatic brain injury. J Neural Transm 2014; 121: 79-90.

47. Tanweer O, Boah A, Huang PP. Risks for hemorrhagic complications after placement of external ventricular drains with early chemical prophylaxis against venous thromboembolisms. J Neurosurg 2013; 119: 1309-1322.

48. Tran LD, Lifshitz J, Witgen BM, Schwarzbach E, Cohen AS, Grady MS. Response of the contralateral hippocampus to lateral fluid percussion brain injury. J Neurotrauma 2006; 23: 1330 1342.

49. Tunç AT, Aslan H, Turgut M, Ekici F, Odaci E, Kaplan S. Inhibitory effect of pinealectomy on the development of cerebellar granule cells in the chick: a stereological study. Brain Res 2007; 1138: 214-234.

50. West MJ, Slomianka L, Gundersen HJ. Unbiased stereological estimation of the total number of neurons in the subdivisions of the rat hippocampus using the optical fractionators. Anat Rec 1991; 231: 482-497.

51. Zilkha-Falb R, Ziv I, Nardi N, Offen D, Melamed E, Barzilai A. Monoamine-induced apoptotic neuronal cell death. Cell Mol Neurobiol 1997; 17: 101-119.

52. Zwienenberg-Lee M, Muizelaar JP. Clinical pathophysiology of traumatic brain injury in humans neurological surgery. HR Saunders, Philadelphia 2004; pp. 5039-5064. 